\title{
Usefulness of Gastric Aspirate Culture for Diagnosing Congenital Immunodeficiency in an Infant with Fungal Pneumonia Caused by Rasamsonia piperina
}

\author{
Yuji Fujita, ${ }^{1,2}$ Naruhiko Ishiwada, ${ }^{3}$ Haruka Takei, ${ }^{1}$ Shin-ichi Suwabe, ${ }^{2}$ \\ Kyoko Yarita, ${ }^{3}$ Misako Ohkusu, ${ }^{3}$ Yasunori Muraosa, ${ }^{3}$ Katsuhiko Kamei ${ }^{3}$ and \\ Naoki Shimojo ${ }^{1}$ \\ ${ }^{1}$ Department of Pediatrics, Chiba University Graduate School of Medicine, Chiba, Chiba, Japan \\ ${ }^{2}$ Department of Pediatrics, Kimitsu Chuo Hospital, Kisarazu, Chiba, Japan \\ ${ }^{3}$ Department of Infectious Diseases, Medical Mycology Research Center, Chiba University, Chiba, Chiba, Japan
}

\begin{abstract}
Chronic granulomatous disease (CGD) is a type of primary immunodeficiency disease, which increases susceptibility to recurrent bacterial and fungal infections. Sputum and bronchoalveolar lavage fluid are often obtained using bronchoscopy from adult patients for pathogenic diagnosis, although this approach is much more invasive for infants. We report the case of a 2-month-old boy with CGD, in which gastric aspirate culture was used to diagnose fungal pneumonia. Rasamsonia piperina was isolated from the gastric aspirate, and the patient was successfully treated with micafungin based on the drug susceptibility test results for the fungal isolate. The acid tolerance test revealed that $R$. piperina could grow at $\mathrm{pH} 2$, indicating high acid resistance. Although we can only report our experience with a single case, gastric aspirate culture may be a useful tool for detecting fungal respiratory pathogens in children with primary immunodeficiency. Detecting these pathogens may help improve outcomes, as early diagnosis and appropriate treatment are extremely important for immunocompromised patients with respiratory infections.
\end{abstract}

Keywords: chronic granulomatous disease; fungal respiratory infection; gastric aspirate; primary immunodeficiency; Rasamsonia piperina

Tohoku J. Exp. Med., 2019 April, 247 (4), 265-269. C 2019 Tohoku University Medical Press

\section{Introduction}

Chronic granulomatous disease (CGD) is a type of primary immunodeficiency disease that is related to defective functioning of nicotinamide adenine dinucleotide phosphate oxidase. Most CGD patients experience recurrent bacterial and fungal infections which can be associated with high rates of morbidity and mortality, although prophylaxis using antibiotics, antifungal agents and interferon- $\gamma$ has reduced the frequency of infections (Marciano et al. 2015). Fungi are especially important pathogenic microorganisms in immunocompromised patients with respiratory infections, and early diagnosis and appropriate treatment are extremely important for improving their prognosis.

Among symptomatic patients, sputum culture is useful for detecting both bacterial and fungal pathogens. Among adult patients with mild respiratory symptoms who may not have readily available sputum samples, bronchial lavage can also be used to obtain the required fluid sample to identify the responsible pathogen. However, it is much more invasive and technically difficult to perform bronchoscopy for children and especially infants. Thus, gastric aspiration may be used to obtain material to diagnose tuberculosis and infection with a yeast-like fungus (Pneumocystis jirovecii) (Chan et al. 1977). This approach is useful in children (Cruz and Strake 2013), although it is unclear whether the gastric aspirate can be used to detect respiratory fungal pathogens. We report the identification of a respiratory fungal pathogen in gastric aspirate from a 2-month-old patient with primary immunodeficiency. The results of the gastric aspirate culture and drug testing were able to support early diagnosis and appropriate treatment of the patient's fungal pneumonia.

\section{Case Presentation}

Informed consent for publishing this case report was obtained from the patient's parents. A 2-month-old boy was suspected of having congenital immune deficiency based on the presence of refractory cervical lymphadenitis. This patient was diagnosed with CGD based on defective neutrophil functioning and a mutation in the $C Y B B$

Received February 15, 2019; revised and accepted April 10, 2019. Published online April 19, 2019; doi: 10.1620/tjem.247.265.

Correspondence: Yuji Fujita, M.D., Department of Pediatrics, Chiba University Graduate School of Medicine, 1-8-1 Inohana, Chuo-ku,

Chiba, Chiba 260-8677, Japan.

e-mail: ojifujita@gmail.com 
(gp91 phox gene. The patient subsequently received prophylactic trimethoprim-sulfamethoxazole and itraconazole (ITCZ) treatment to prevent bacterial and fungal infections. Although the patient only had mild respiratory signs, chest computed tomography (CT) was performed to screen for respiratory infections and revealed several nodules in the right lung (Fig. 1A). This finding supported a suspicion of fungal infections, and the nodules increased in size over the following month (Fig. 1B). The patient also had an increased serum concentration of Creactive protein but was negative for various serum fungal markers ( $\beta$-D-glucan and antigens from Aspergillus spp. and Candida spp.).

Because we could not obtain a sputum sample, gastric aspirate was collected and cultured to detect fungi and mycobacteria. After several days, filamentous fungus had started to grow on Sabouraud dextrose agar plates. Sequences of the internal transcribed spacer regions of the ribosomal RNA gene were used to identify the fungus as Rasamsonia piperina, and an isolate was submitted to the Medical Mycology Research Center at Chiba University (strain no. IFM 65240). Antifungal drug susceptibility testing revealed that the isolate had high minimum inhibitory concentrations (MICs) for voriconazole (VRCZ, $>8 \mathrm{mg} / \mathrm{L})$ and amphotericin B $(2 \mathrm{mg} / \mathrm{L})$ but low MICs for ITCZ $(0.5 \mathrm{mg} / \mathrm{L})$, micafungin (MCFG, $<0.015 \mathrm{mg} / \mathrm{L}$ ), and flucytosine $(<0.12 \mathrm{mg} / \mathrm{L})$. The patient was treated using intravenous MCFG for 8 weeks and then using oral flucytosine for 4 weeks. The patient exhibited clinical improvement after the MCFG treatment, and chest CT revealed that the lung nodules had gradually regressed (Fig. 1C, D). However, we were worried that the nodules had grown during the prophylactic ITCZ treatment, despite the in vitro test results indicating that the isolate was susceptible to ITCZ. We, therefore, measured the patient's serum concentration of ITCZ, which revealed that the serum trough concentration of ITCZ $(42.5 \mathrm{ng} / \mathrm{mL})$ was much lower than the target concentration $(>500 \mathrm{ng} / \mathrm{mL}$ ).

The $R$. piperina isolate's acid resistance was tested by comparing its growth to that of Aspergillus fumigatus (IFM 59355). The conidia were inoculated into Czapek-Dox broth media, which were adjusted to $\mathrm{pH}$ readings of 6.0-1.0 and then incubated at $37^{\circ} \mathrm{C}$ for $48 \mathrm{~h}$. The results revealed that the $R$. piperina isolate grew at a $\mathrm{pH}$ value of 2 and $A$. fumigatus grew at a $\mathrm{pH}$ value of 3 , which indicated that both fungi had high acid resistance (Fig. 2).

\section{Informed consent}

Informed consent for publishing this case report was obtained from the patient's parents.

\section{Discussion}

Detection of pathogenic fungi is extremely important for the appropriate treatment of respiratory infections. Serological testing for $\beta$-D-glucan and some fungal antigens may be useful (De Pauw et al. 2008), although not all cases

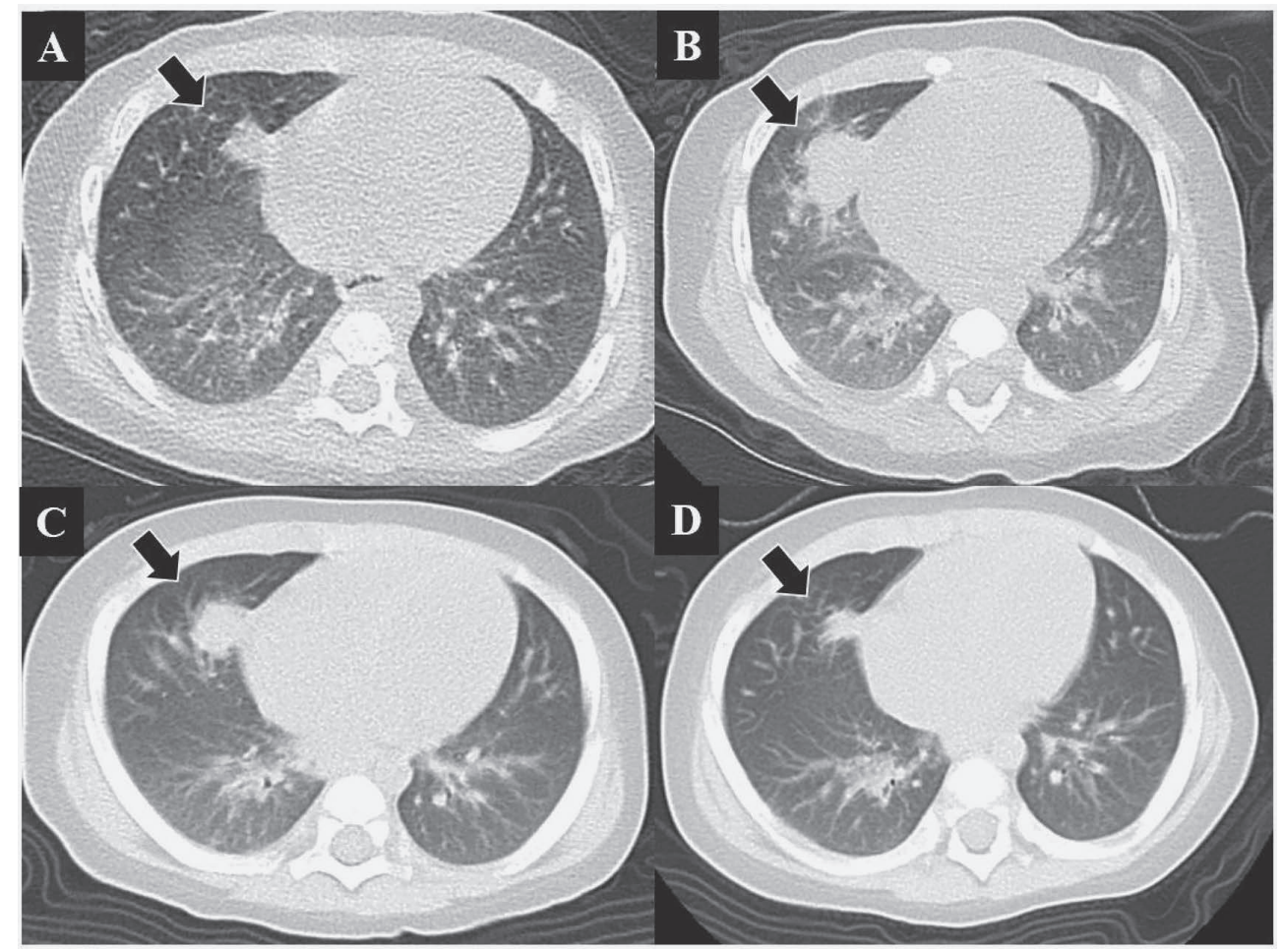

Fig. 1. Chest computed tomography results.

Chest computed tomography revealed growth of nodules in the right lung before treatment, with gradual regression after antifungal treatment. Results are shown from (A) at the diagnosis of chronic granulomatous disease, (B) before treatment, (C) 1 month after starting treatment, and (D) 3 months after starting treatment. 
Acid tolerance test

Aspergillus fumigatus

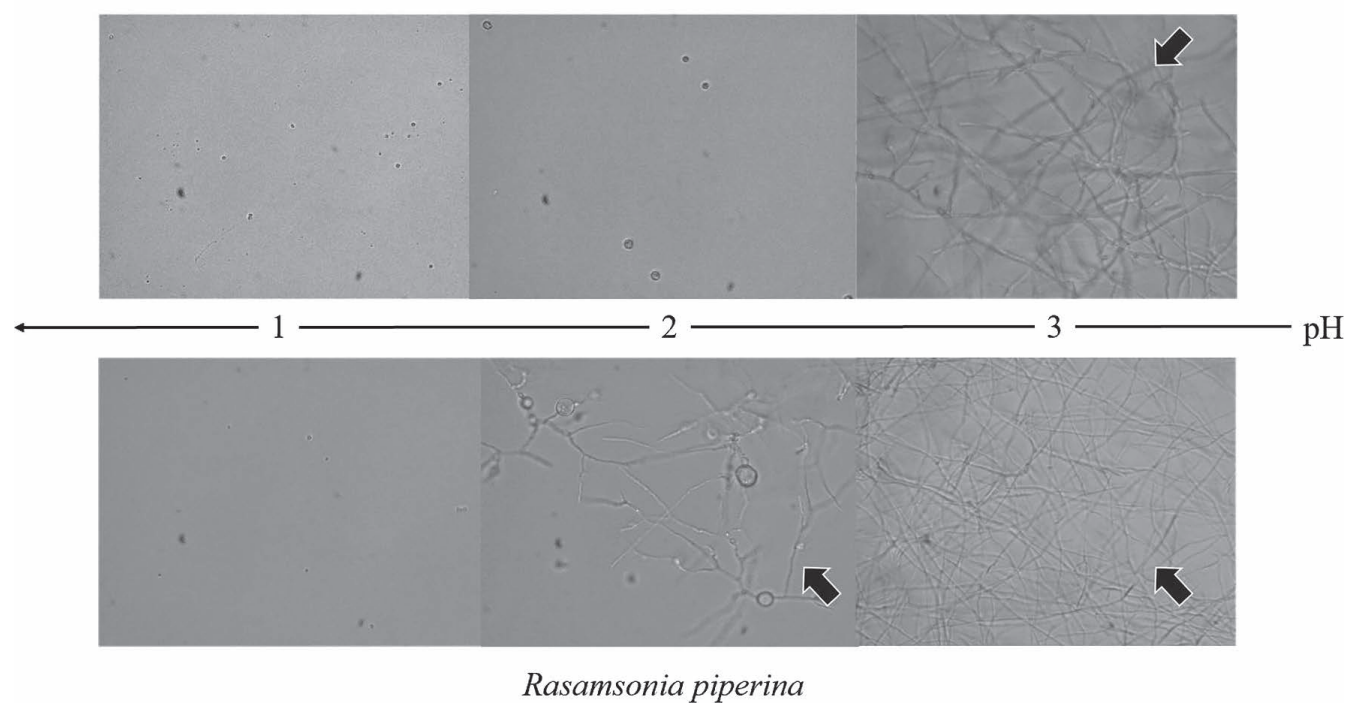

Fig. 2. Acid tolerance test results.

The acid tolerance test revealed that the R. piperina isolate grew at $\mathrm{pH} 2$ and A. fumigatus grew at $\mathrm{pH} 3$.

have elevated $\beta$-D-glucan concentrations and the available antigens are limited for Aspergillus spp., Candida spp., and Cryptococcus spp. Thus, culture is the gold standard for the diagnosis of fungal infections, with sputum typically being suitable for detecting respiratory fungal pathogens, especially filamentous fungi. However, sputum samples are difficult to obtain from asymptomatic children, and bronchoscopy to obtain bronchoalveolar lavage fluid is much more invasive for pediatric patients than for adult patients. Therefore, other methods are needed to detect respiratory fungal pathogens in children.

Gastric aspirate has been used to detect several respiratory pathogens in children, as the children swallow bronchial secretions while sleeping and acid-resistant microorganisms can be detected in their gastric aspirate (Rigouts 2009). For example, gastric aspirate can be used to diagnose tuberculosis in children, and other reports have described using gastric aspirate to detect fungi, including Pneumocystis jirovecii and Blastomyces dermatitidis, in children (Chan et al. 1977; Fanella et al. 2010). Thus, gastric aspirate may be useful for detecting respiratory fungal pathogens. Our patient suffered from CGD, which elevates the risk of fungal infection, and the $\mathrm{CT}$ results supported a suspicion of a fungal infection, despite the patient only having mild respiratory signs. Accordingly, to avoid more invasive testing methods, we attempted to detect the pathogen using the patient's gastric aspirate.

The present case involved an isolate of $R$. piperina, which was originally known as Geosmithia argillacea before being transferred to the Rasamsonia genus (Houbraken et al. 2012). An initial report indicated that Geosmithia argillacea caused invasive mycosis in an adult patient with CGD (De Ravin et al. 2011), and Ishiwada et al. (2016) have reported a patient with CGD and osteomyelitis caused by $R$. piperina, which was successfully treated with MCFG. Similar to our findings, these previous reports have indicated that $R$. piperina is often resistant to VRCZ and posaconazole but is susceptible to MCFG.

The acid tolerance test revealed that $R$. piperina isolate and A. fumigatus could both grow at $\mathrm{pH} 3$. Importantly, younger children have weaker gastric acid secretion than older children (Lucas et al. 1982; Boyle 2003). Furthermore, it has been reported that bacterial growth is enhanced under gastric secretion inhibitor treatment (Ruddell et al. 1980), and some fungi may also grow in acidic environments (Yamazaki et al. 2010). Moreover, this technique may be useful in patients receiving proton pump inhibitors, which decrease gastric acid secretion. Thus, future research should examine whether this technique is useful for detecting other fungi.

Our patient's fungal pneumonia had worsened during the prophylactic ITCZ treatment, which may have been influenced by the patient's immune status, the prophylactic antifungal treatment, and environmental issues. In this context, CGD is the most common type of primary immunodeficiency, which is associated with increased susceptibility to fungal infection, and children with CGD typically receive prophylactic antifungal treatment. Prophylaxis using ITCZ reduces the risk of fungal infection (Gallin et al. 2003), especially with Aspergillus spp. and Candida spp., although the pharmacokinetics of ITCZ in infants remain unclear. In the present case, the $R$. piperina isolate had a low MIC for ITCZ, although the serum concentration of ITCZ was also low and likely explains the progression of the R. piperina infection. Moreover, there is inter-patient variability in the absorption of ITCZ, which 
highlights the importance of monitoring and maintaining optimal serum concentrations (Mofenson et al. 2009). It is also necessary to consider the appropriate dosage of ITCZ for children based on environmental factors. In this case, the patient had been living in a house with large amounts of mold, and he may have inhaled a relatively large amount of fungus, although we were unable to perform a direct environmental investigation. Controlling exposure to fungus (e.g., by cleaning or moving) may help limit the inhaled amount of fungus.

It is important to identify the causative fungal pathogen to guide appropriate antifungal treatment, especially for species that are resistant to ITCZ (Salvator et al. 2015). Although many of these fungi are susceptibility to VRCZ and posaconazole (Segal et al. 2005; Alsultan et al. 2006), some species may be resistant to all of these antifungal agents. In the present case, the in vitro test allowed us to select MCFG treatment over VRCZ treatment, based on the MICs, which led to improvement of the fungal pneumonia. Thus, the present case further highlights the importance of testing to identify the causal pathogen and to determine its anti-fungal drug sensitivity.

In conclusion, gastric aspiration may be a useful tool for detecting respiratory fungal pathogens in children, especially infants, with primary immunodeficiency. However, this report only describes our experience with a single case, and additional research is needed to validate this technique.

\section{Acknowledgments}

This report was funded by the Project for the development of prevention, diagnosis and treatment against elderly and neonatal aspergillosis, Chiba University.

\section{Author Contributions}

Dr. Fujita drafted and revised the initial manuscript. Dr. Ishiwada suggested using gastric aspirate to detect fungal pathogens and supervised the writing of the manuscript. Dr. Haruka, Dr. Suwabe, Prof. Kamei, and Prof. Shimojo critically revised the manuscript for important intellectual content. Dr. Yarita and Dr. Ohkusu performed the fungal identification and the drug sensitivity testing. Dr. Muraosa performed the fungal acid tolerance test and interpreted the results. All authors approved the final manuscript and agree to be accountable for all aspects of the work. All authors meet the ICMJE authorship criteria.

\section{Conflict of Interest}

The authors declare no conflict of interest.

\section{References}

Alsultan, A., Williams, M.S., Lubner, S. \& Goldman, F.D. (2006) Chronic granulomatous disease presenting with disseminated intracranial aspergillosis. Pediatr. Blood Cancer, 47, 107-110.

Boyle, J.T. (2003) Acid secretion from birth to adulthood. J. Pediatr. Gastroenterol. Nutr., 37 Suppl 1, S12-16.

Chan, H., Pifer, L., Hughes, W.T., Feldman, S., Pearson, T.A. \& Woods, D. (1977) Comparison of gastric contents to pulmonary aspirates for the cytologic diagnosis of Pneuomcystis carinii pneumonia. J. Pediatr., 90, 243-244.

Cruz, A.T. \& Strake, J.R. (2013) TUBERCULOSIS. In Feigin and Cherry's Textbook of Pediatric Infections and Diseases, 7th ed., edited by Cherry, J., Demmler-Harrison, G., Kaplan, S., Steinbach, W. \& Hotez, P. Elsevier, Philadelphia, PA, pp. 1364-1365.

De Pauw, B., Walsh, T.J., Donnelly, J.P., Stevens, D.A., Edwards, J.E., Calandra, T., Pappas, P.G., Maertens, J., Lortholary, O., Kauffman, C.A., Denning, D.W., Patterson, T.F., Maschmeyer, G., Bille, J., Dismukes, W.E., et al. (2008) Revised definitions of invasive fungal disease from the European Organization for Research and Treatment of Cancer/Invasive Fungal Infections Cooperative Group and the National Institute of Allergy and Infectious Diseases Mycoses Study Group (EORTC/MSG) Consensus Group. Clin. Infect. Dis., 46, 1813-1821.

De Ravin, S.S., Challipalli, M., Anderson, V., Shea, Y.R., Marciano, B., Hilligoss, D., Marquesen, M., Decastro, R., Liu, Y.C., Sutton, D.A., Wickes, B.L., Kammeyer, P.L., Sigler, L., Sullivan, K., Kang, E.M., et al. (2011) Geosmithia argillacea: an emerging cause of invasive mycosis in human chronic granulomatous disease. Clin. Infect. Dis., 52, e136-143.

Fanella, S., Walkty, A., Bridger, N., Crockett, M., ConsunjiAraneta, R., Embree, J. \& Karlowsky, J. (2010) Gastric lavage for the diagnosis of pulmonary blastomycosis in pediatric patients. Pediatr. Infect. Dis. J., 29, 1146-1148.

Gallin, J.I., Alling, D.W., Malech, H.L., Wesley, R., Koziol, D., Marciano, B., Eisenstein, E.M., Turner, M.L., DeCarlo, E.S., Starling, J.M. \& Holland, S.M. (2003) Itraconazole to prevent fungal infections in chronic granulomatous disease. N. Engl. J. Med., 348, 2416-2422.

Houbraken, J., Spierenburg, H. \& Frisvad, J.C. (2012) Rasamsonia, a new genus comprising thermotolerant and thermophilic Talaromyces and Geosmithia species. Antonie Van Leeuwenhoek, 101, 403-421.

Ishiwada, N., Takeshita, K., Yaguchi, T., Nagasawa, K., Takeuchi, N., Hishiki, H., Watanabe, A., Kamei, K. \& Shimojo, N. (2016) The first case of invasive mixed-mold infections due to Emericella nidulans var. echinulata and Rasamsonia piperina in a patient with chronic granulomatous disease. Mycopathologia, 181, 305-309.

Lucas, A., Bloom, S.R. \& Aynsley-Green, A. (1982) Postnatal surges in plasma gut hormones in term and preterm infants. Biol. Neonate, 41, 63-67.

Marciano, B.E., Spalding, C., Fitzgerald, A., Mann, D., Brown, T., Osgood, S., Yockey, L., Darnell, D.N., Barnhart, L., Daub, J., Boris, L., Rump, A.P., Anderson, V.L., Haney, C., Kuhns, D.B., et al. (2015) Common severe infections in chronic granulomatous disease. Clin. Infect. Dis., 60, 1176-1183.

Mofenson, L.M., Brady, M.T., Danner, S.P., Dominguez, K.L., Hazra, R., Handelsman, E., Havens, P., Nesheim, S., Read, J.S., Serchuck, L. \& Van Dyke, R.; Centers for Disease Contro and Prevention; National Institutes of Health; HIV Association of the Infectious Diseaes Society of America; Pediatric Infectious Diseaes Society; American Academy of Pediatrics (2009) Guidelines for the prevention and treatment of opportunistic infections among HIV-exposed and HIV-infected children: recommendations from CDC, the National Institutes of Health, the HIV Medicine Association of the Infectious Diseases Society of America, the Pediatric Infectious Diseases Society, and the American Academy of Pediatrics. MMWR Recomm. Rep., 58, 1-166.

Rigouts, L. (2009) Clinical practice: diagnosis of childhood tuberculosis. Eur. J. Pediatr., 168, 1285-1290.

Ruddell, W.S., Axon, A.T., Findlay, J.M., Bartholomew, B.A. \& Hill, M.J. (1980) Effect of cimetidine on the gastric bacterial flora. Lancet, 1, 672-674.

Salvator, H., Mahlaoui, N., Catherinot, E., Rivaud, E., Pilmis, B., Borie, R., Crestani, B., Tcherakian, C., Suarez, F., Dunogue, B., Gougerot-Pocidalo, M.A., Hurtado-Nedelec, M., Dreyfus, 
J.F., Durieu, I., Fouyssac, F., et al. (2015) Pulmonary manifestations in adult patients with chronic granulomatous disease. Eur. Respir. J., 45, 1613-1623.

Segal, B.H., Barnhart, L.A., Anderson, V.L., Walsh, T.J., Malech, H.L. \& Holland, S.M. (2005) Posaconazole as salvage therapy in patients with chronic granulomatous disease and invasive filamentous fungal infection. Clin. Infect. Dis., 40, 16841688.

Yamazaki, A., Toyama, K. \& Nakagiri, A. (2010) A new acidophilic fungus Teratosphaeria acidotherma (Capnodiales, Ascomycota) from a hot spring. Mycoscience, 51, 443-455. 